\title{
Analysis of Leach and Improved Leach Protocol in Wireless Sensor Network
}

\author{
Harpreet Kaur ${ }^{1}$, Amit Verma ${ }^{2}$, Manit Kapoor ${ }^{3}$ \\ Student, ECE, Ramgarhia Institute of Engineering and Technology, Phagwara, India ${ }^{1}$ \\ Assistant Professor, ECE, Ramgarhia Institute of Engineering and Technology, Phagwara, India ${ }^{2,3}$
}

\begin{abstract}
This paper describes energy-efficient communication in wireless Sensor Networks is necessary and identifies the main sources of energy dissipation as well as counter measures to make sure a long network lifetime. Wireless Sensor Networks can collect trusty and accurate information in distant and risky environments, and can be used in National Defence, Military Affairs, Industrial Control, Traffic Management, Medical Care, Smart Home, etc. The sensor whose resources are reserved is low-cost, and depends on battery to supply electricity, so it's importance for routing to efficiently utilize its power. The main purpose of this Research work is to investigate and find insight into the effect of energy efficient for wireless Sensor Networks using Leach and proposed new Leach protocol and their parameter. As noted, the research consists of both experimental and analytical results on MATLAB tool with the following parameter average energy of each node for different- different no of rounds, number of dead nodes, total number of packet received to base station and end to end delay.
\end{abstract}

Keywords: WSN, LEACH, Proposed LEACH, Network lifetime.

\section{INTRODUCTION}

The Wireless sensor network is composed of a cluster of rotation mechanism, data aggregation, and data fusion sensor nodes, which are small-scale energy constrained technologies effectively improves the lifetime of a devices. The efficient use of energy source in a sensor network. In order to improve energy in the network, nodes node is most adorable criteria to improve the life time of are selected as cluster head circularly and randomly. The wireless sensor network. Wireless sensor networks normal nodes called cluster members join the advancement is expeditiously increasing and that's why corresponding cluster head nodes on the basis of principle there is an immense field for research in this area. So the of proximity. Normal nodes sense data and send directly to design of energy aware routing protocol is essential in the cluster head nodes. The cluster head nodes receive order to enhance the network lifetime and to mitigate sensed data, aggregate the data to remove redundancy and power losses. Therefore, to optimize network lifetime and fusion processes are carried out and data is send to the sink achieve high reliability and scalability, routing techniques (or Base Station). So LEACH increases network lifetime have been developed. LEACH is the conventional by decreasing network energy consumption, and reducing hierarchical clustering protocol traditionally used in number of communication messages by data aggregation wireless network. LEACH is energy-efficient hierarchical and fusion. The process of formation of clusters in based routing protocol that balances the energy expense, LEACH is shown in figure 1.

saves the node energy and hence prolongs the network lifetime. In this paper we design a new proposed protocol which is based on the LEACH protocol. This proposed routing protocol consists of five different levels of energy nodes. These nodes are named as ultra nodes, super nodes, advance nodes, intermediate nodes, and normal nodes. This routing protocol is threshold sensitive. In the Performance evaluation of existing and proposed protocol on the basis of network lifetime we measure proposed protocol gives better results on same input parameters.

\section{CLUSTER BASED HIERARCHICAL ROUTING PROTOCOL}

Low-Energy Adaptive Clustering Hierarchy (LEACH)] is a essential hierarchical clustering routing protocol, which accept distributed clustering algorithm where cluster-head

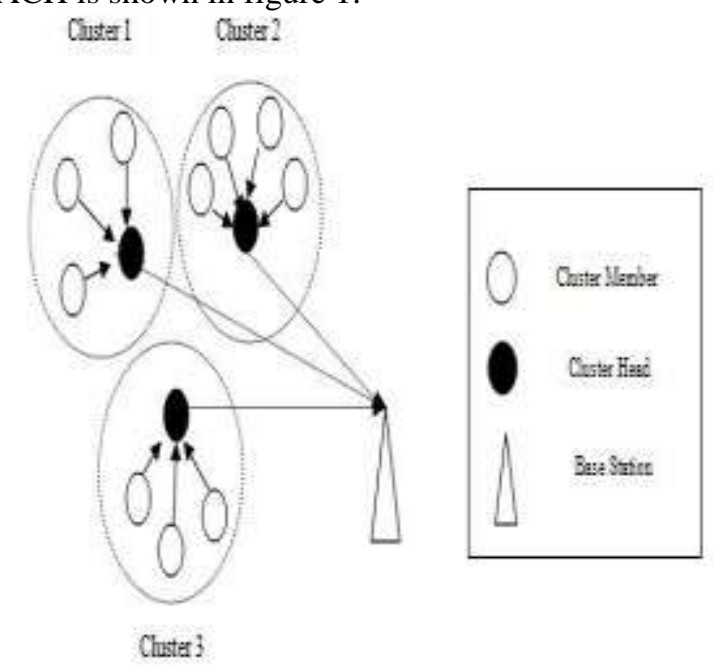

Fig. 1: Formation of Clusters in LEACH 
In order to achieve the design goal the key tasks performed by Leach are as follows:

$>$ Randomized rotation of the cluster heads and the corresponding clusters.

$>$ Global communication reduction by the local compression.

$>$ Localized co-ordination and control for cluster setup and operation.

$>$ Low energy media access control.

$>$ Application specific data processing.

\section{DEFICIENCIES IN CLASSICAL LEACH PROTOCOL}

\section{A. Unreasonable cluster head selection}

LEACH protocol doesn't take residual energy of each node into consideration for the selection of cluster head node as each node has equal probability of becoming cluster head. If low-energy node is being selected as cluster head node, then the network fails soon due to high energy consumption causes adverse to energy balancing among the network. This results data loss and lower in survival time of the network.

\section{B. Unreasonable distribution of cluster heads}

The random selection algorithm of cluster head nodes causes problem of imbalance in energy load. Distance factor is not considered in cluster formation due to which sometimes very big clusters and very class clusters exist at the same time in the network. More the distance between cluster head node and base station, more the energy consumption of that node.

\section{More responsibility on Cluster Head node}

Cluster head nodes perform data aggregation and send processed data to the base station in single-hop due to which cluster head nodes deplete their energy too fast as compared to normal nodes. Also if a cluster head node fails, the whole nodes linked to it will deplete their energy too.

\section{IV.PROPOSED WORK}

Efficiently use of energy in the network has been the main issue in Wireless sensor network for prolonging lifetime of the network. LEACH has found one of the most energy efficient protocol used in Wireless sensor network. In Wireless Sensor Networks the main purpose of designing energy efficient routing protocol is to efficiently use the energy of the network so that the network lifetime get increased.

In WSNs various energy efficient routing protocols are available now-a-days. One of the most energetic routing algorithms everyone uses is the LEACH routing protocol. . In this research work, LEACH protocol has been discussed with its drawbacks and how these drawbacks are overcome by its descendants. A brief study of various improved versions of LEACH protocol has been done in order to compare performance of these descendants with the classical LEACH. It is concluded from given survey that for prolonging network lifetime of WSN, there is need to explore more robust, reliable and efficient protocols in future.

In present work I have implemented the five different level of energy node. Routing protocol which consists, five different level of energy nodes: Ultra nodes, Super nodes, Advance nodes, Intermediate nodes, Normal nodes. LEACH protocol to improve the network lifetime. To compare the LEACH protocol with Proposed LEACH protocol in the basis of average energy of the nodes and network lifetime of nodes. The Improved LEACH is more effective then the LEACH. In LEACH network lifetime of the nodes is very less as compared to Proposed LEACH and average energy decrease shortly as compared to Proposed LEACH.

\section{PARAMETERS USED}

TABLE 1 Parameters used

\begin{tabular}{|c|c|c|}
\hline Parameters & LEACH & Proposed Leach \\
\hline No. of Nodes & 100 & 100 \\
\hline $\begin{array}{c}\text { Probability of } \\
\text { cluster heads P }\end{array}$ & 0.1 & 0.1 \\
\hline Initial energy Eo & .5 & .5 \\
\hline $\begin{array}{c}\text { Transmit energy } \\
\text { ETX }\end{array}$ & $\begin{array}{c}50 * 0.00000 \\
0001\end{array}$ & $50 * 0.00000000$ \\
\hline Receive energy & $50 * 0.00000$ & $50 * 0.00000000$ \\
RTX & 0001 & 1 \\
\hline $\begin{array}{c}\text { Free space loss } \\
\text { EFS }\end{array}$ & $\begin{array}{c}10 * 0.00000 \\
000001\end{array}$ & $10 * 0.00000000$ \\
001 \\
\hline $\begin{array}{c}\text { Multi path loss } \\
\text { EMP }\end{array}$ & $0.0013 * 0.00$ & $0.0013 * 0.00000$ \\
0000000001 & 0000001 \\
\hline $\begin{array}{c}\text { Data aggregation } \\
\text { energy EDA }\end{array}$ & $50 * 0.00000$ & $50 * 0.00000000$ \\
& 0001 & 1 \\
\hline Maximum & 5000 & 10000 \\
number of rounds & & \\
\hline \multicolumn{2}{|c|}{} \\
\hline
\end{tabular}

\section{VI.RESULT AND DISCUSSION}

In figure 2 firstly we calculate the alive node by using LEACH protocol represented by the "red line". And secondly we calculate the alive node by using proposed LEACH protocol represented by the" blue line". Result of both algorithms combined on one figure. This algorithm minimizes the amount of energy for non cluster head nodes to transmit their data to the cluster head nodes by minimizing the distance between all cluster head nodes and non cluster head nodes. BS considers some nodes as $\mathrm{CHs}$ and Non CHs. BS broadcasts a message of cluster head IDs to all nodes. If node ID is same as cluster head ID, is selected as cluster head and determine TDMA schedule for data transfer. 


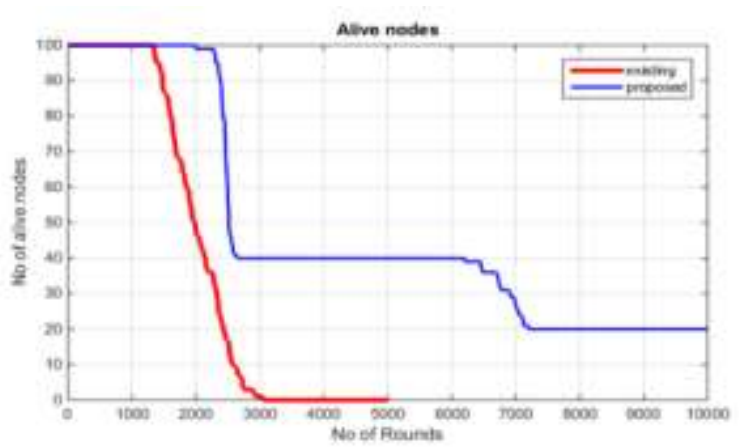

Fig. 2 Comparison of no. of alive nodes

TABLE 2 Comparison of alive nodes

\begin{tabular}{|c|c|c|c|}
\hline \multicolumn{2}{|c|}{ No of Alive Nodes } & \multicolumn{2}{c|}{ No. of rounds } \\
\hline LEACH & Proposed LEACH & LEACH & Proposed LEACH \\
\hline 100 & 100 & 1300 & 2220 \\
\hline 90 & 90 & 1500 & 2400 \\
\hline 80 & 80 & 1650 & 2500 \\
\hline 70 & 70 & 1750 & 2530 \\
\hline 60 & 60 & 1900 & 2550 \\
\hline 50 & 50 & 1900 & 2570 \\
\hline 40 & 40 & 2200 & 5000 \\
\hline 30 & 30 & 2400 & 7000 \\
\hline 20 & 20 & 2500 & 8000 \\
\hline 10 & 10 & 2650 & 8050 \\
\hline 0 & 0 & 3000 & 8100 \\
\hline
\end{tabular}

Figure 3 presents the number of control packets versus the number of rounds for the protocols. It clearly seen that the Proposed LEACH protocol have more control packets because five different level of energy nodes. Proposed LEACH has more control packets are broadcast. Proposed LEACH has each node sends information the best cluster head and broad casts a message that obtains the cluster head ID for each node which leads more control packets.

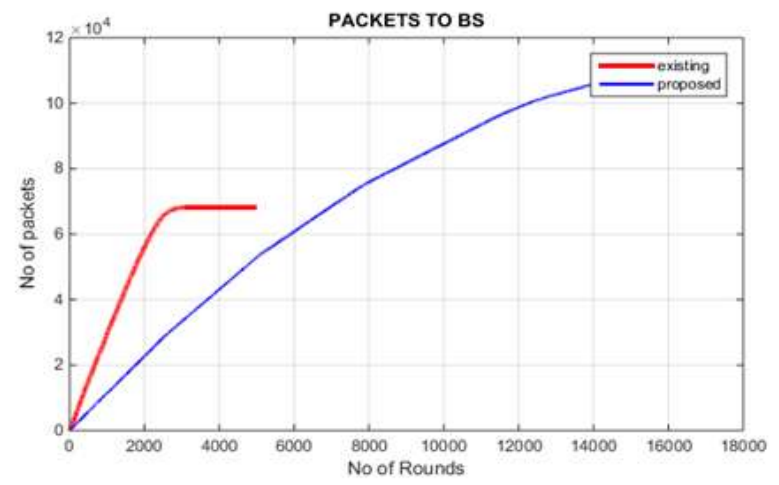

Fig. 3 Comparison of the control of packets

TABLE 3 Comparison of the control of packets

\begin{tabular}{|c|c|c|}
\hline No of Packets & \multicolumn{2}{|c|}{ No of Rounds } \\
\hline & LEACH & Proposed LEACH \\
\hline $2 * 10000$ & 900 & 2000 \\
\hline $4 * 10000$ & 1500 & 4000 \\
\hline
\end{tabular}

\begin{tabular}{|c|c|c|}
\hline $6 * 10000$ & 2100 & 6000 \\
\hline $8 * 10000$ & & 8000 \\
\hline $10 * 1000$ & & 12000 \\
\hline
\end{tabular}

In figure 4 "Red line" are declared as LEACH nodes. On other side "Blue line" are declared as Proposed LEACH nodes.

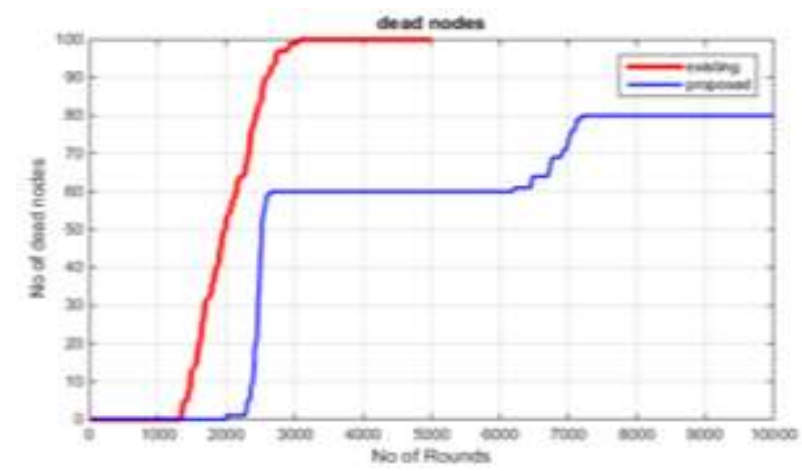

Fig. 4 Comparison of LEACH and Proposed LEACH on the basis of dead nodes

\section{CONCLUSION}

In this paper we study the LEACH protocol and design a new proposed protocol which is based on the LEACH protocol. This proposed routing protocol consists of five different levels of energy nodes. These nodes are named as ultra nodes, super nodes, advance nodes, intermediate nodes, and normal nodes. This routing protocol is threshold sensitive. In the performance evaluation of existing and proposed protocol on the basis of network lifetime we measure proposed protocol gives better results on same input parameters.

\section{REFERENCES}

1. VivekKatiyar, Narottam Chand, SurenderSoni (2010) "Clustering Algorithm for Heterogeneous Wireless Sensor Network: A Survey", International journal of applied engineering research.

2. S.K. Ghosh, M.K. Ghose and Kalpana Sharma(2010) "Establishing an integrated secure Wireless Sensor Network System: A New Approach" International Journal of next-Generation Networks.

3. Liyang Yu, Neng Wang and XiaoqiaoMeng, "Real-time Fire detection with wireless sensor Networks" East China University Shanghai, China 2011.

4. YunusEmreAslan, Ibrahim Korpeolu. OzgurUlusoy (2012) “A framework for use of wireless sensor networks in fire detection and Monitoring",Bilkent university, department of computer engineering

5. W. R. Heinzelman, A. Chandrakasan, and H. Balakrishnan"Energy Efficient Communication Protocol for Wireless Micro Sensor Networks,"Proceedings of IEEE HICSS Jan 2000.

6. T. Meng and R. Volkan. Distributed Network Protocols for Wireless Communication. In Proc. IEEEE ISCAS, May 1998.

7. O. Younis and S. Fahmy, "HEED: A Hybrid Energy-Efficient Distributed Clustering Approach for Ad Hoc Sensor Networks,"

8. V. Loscri, G. Morabito, and S. Marano, A Two-Level Hierarchy for Low-Energy Adaptive Clustering Hierarchy, DEIS Department, University of Calabria.

9. S. Bandyopadhyay and E. J. Coyle, "An Energy Efficient HierarchicalClustering Algorithm for Wireless Sensor Networks," IEEE INFOCOM, April 2003. 